\title{
Research on Comprehensive Evaluation System for 220/110 kV Electromagnetic Looped Network Operation Modes
}

\author{
He Yu-Qing \\ Hunan Electric Power Corporation Economical \& \\ Technical Research Institute \\ Changsha, Hunan Province, China \\ e-mail: iheyuqing@163.com
}

He Yuan-Yuan

Chenzhou Electric Power Corporation Economical \& Technical Research Centre

Chenzhou, Hunan Province, China

e-mail: iheyuanyuan@163.com

\author{
Mao Li-Lin \\ Hunan electric power transmission and transformation \\ investigation design and Consulting Co. Ltd. \\ Changsha, Hunan Province, China \\ e-mail:maolilin@126.com \\ $\mathrm{Xu}$ Chao \\ Hunan Electric Power Corporation Economical \& \\ Technical Research Institute \\ Changsha, Hunan Province, China \\ e-mail:xuchao@163.com
}

\begin{abstract}
Indiscriminate considering all 220/110 kV electromagnetic looped networks to be opened may affect the power system security, reliability and economical efficiency adversely. This paper focuses on this very issue and discusses the operation mode regarding on the circumstance under which looped network shall be opened or closed. A comprehensive evaluation system is put forward in this paper. Also the specific content of each index, evaluation basis and principles are discussed. The evaluation indexes are divided into two major categories as rigid type and elastic type, and the evaluation method of the later one is proposed. The evaluation process for $220 / 110 \mathrm{kV}$ electromagnetic loop operation mode decision is illustrated. The optimization result and impact of the operation mode to all the 220/110 $\mathrm{kV}$ electromagnetic loop in Hunan power system are analyzed. Based on the actual $220 / 110 \mathrm{kV}$ electromagnetic loops operating cases in Hunan Province, feasibility and superiority for certain 220/110 kV electromagnetic loops being closed is verified.
\end{abstract}

Keywords- electromagnetic looped network; operation modes; opened-loop mode; closed-loop mode; evaluation system

\section{INTRODUCTION}

The electromagnetic loop network is the annular network consisted of two voltage level transmission lines which are connected through electromagnetic coupling namely transformers during the power system development. It has been studied that during certain operation modes, the looped network has stringent impacts on system transmission limit, thermal stability, protection regime and short circuit current level, etc ${ }^{[1-2]}$. Due to the aforementioned issues, the majority of $220 / 110 \mathrm{kV}$ electromagnetic loops are operated in open mode in China. Nowadays electromagnetic loops of higher voltage level are gradually opened with the network development. The current researches mainly focus on how to optimize the opening process ${ }^{[3-11]}$.

Nevertheless, the opened $220 / 110 \mathrm{kV}$ electromagnetic loop is a double-edged sword. On one hand, it reduces damage to the system security due to serious faults on $220 \mathrm{kV}$ networks which arouse the need for power flow transfer. On the other hand it decreases the stability margin and power supply reliability of partial regional. It is difficult to guarantee the network security under openedloop mode operation, especially for regional weak network areas. However, with the daily refined requirements on grid management, indiscriminate considering all 220/110 $\mathrm{kV}$ electromagnetic loops to be opened may affect the power system security, reliability and economical efficiency adversely which manifests following aspects:

1) Regional grid run in parallel with main grid issue: As the electromagnetic loops between regional grids and the mains are gradually opened, the number of tied points is reduced. Following a disturbance, it may lead to disconnection between main supply and the local grid, thus the local network would suffer from severe frequency drop or over frequency damage. Though such issues can be resolved through automatic devices such as load-shedding devices or generator-trip devices, if the automatic devices failed to response correctly, the local grid may collapse due to the abnormal frequency in case of accident.

2) Single power supply issue: Following the electromagnetic loop opening, certain low voltage substations (i.e. $110 \mathrm{kV}$ and below) may become single power sourced which reduces the reliability of network eventually. Even with the installed automatic devices, some loads are still shed from the main due to the low voltage and causes economic loss. If maloperation happened to the automatic devices, malignant loss-of-main accident of the whole substation could be triggered. 
3) Grid economic operation issue: The power flow within the network is redistributed after opening electromagnetic loop. In some cases, the network loss will elevate, particularly for those lines from sources sending power out will overloaded and line losses become extreme high. The rise of line losses results in economic loss as well.

4) Investment pressure issue: In order to improve the reliability of the power grid, it is required to advance the bill for $220 \mathrm{kV}$ substation expansion or build a new substation to satisfy $\mathrm{N}-1$ security check. It leads to increased one-time investment and financial pressure due to debt service.

From the above analysis, the impact of electromagnetic loop is relative rather than definite in respect of system secure and economic operation. Therefore whether to open the loop or not should take security, stability, reliability, economy and other aspects into full consideration. In this paper, a comprehensive evaluation system based on group of evaluation indexes is put forward. The evaluation indexes are divided into two major categories as rigid type and elastic type. The evaluation process for $220 / 110 \mathrm{kV}$ electromagnetic loop operation mode decision is illustrated. Taking Hunan power grid as an example, the $220 / 110 \mathrm{kV}$ electromagnetic loop operation modes optimization results and effect are analyzed. Combined with the actual closed operated electromagnetic loop in Hunan network, feasibility and superiority for certain 220/110 kV electromagnetic loops being closed is verified.

\section{EVALUATION INDEXES SYSTEM}

\section{A. Evaluation indexes}

220/110kV electromagnetic loop evaluation indexes are divided into two major categories as rigid type and elastic type. The rigid indexes must be satisfied which include short circuit current level, power system static security, transient stability and implemented environment while the static security index considering two aspects: N1 security level and stability margin. The elastic indexes include reliability level and comprehensive economic efficiency while the economic indexes consist of technical reform investment and loss reduction. The index system frame is shown in Fig .1.

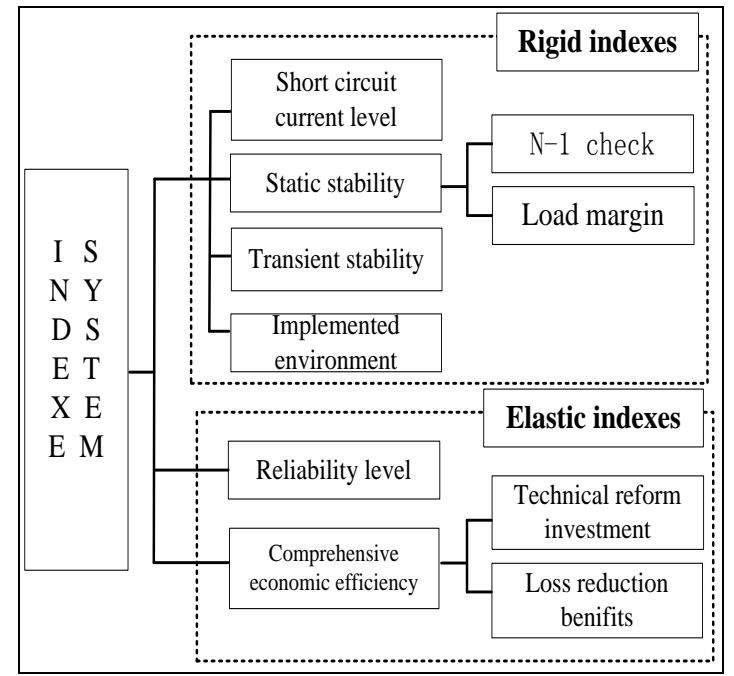

Figure 1. Index system

\section{B. Indexes connotation}

\section{1) Rigid indexes}

\section{a) Short circuit level}

When short circuit occurs in the power system, large short circuit current is generated with electric arc. It not only burns down the fault device itself but also jeopardizes the devices and persons around. Large current through conductors at one hand can cause rapid buildup of heat, potentially result in damage to the insulation, or even meltdown. On the other hand large current produces great electrodynamic force which deforms and damages the conductors. It also causes system voltage slash, especially near the fault point where voltage reduces more, which may cause power supply interruption to part or all users. Given the harms of short circuit current, either adopting opened loop or closed loop operation, the short circuit current for each voltage level should be limited within the rated breaking capacity of the circuit breaker with appropriate margin. This paper sets $10 \%$ of the rated breaking capacity to be the margin value during later evaluation process.

\section{b) System static security}

Closed-loop operation shall meet the N-1 security level requirement without decreasing the load margin. At present, the main concern about closed-loop mode lays on two aspects. First of all, within a closed electromagnetic loop, once a higher level voltage lines is tripped, the impedance of the tie line would suddenly increases significantly, the power flow through the original high voltage transmission line will be transferred to some or all of low voltage lines in parallel. This will lead to line overloads and transformers overloads. It can also cause power flow through the low voltage lines to exceed their stability limit or even thermal stability limit. Secondly the stability limit of the closed-loop operated network is reduced and may cause system oscillation.

c) System transient stability

Under closed 220/110kV electromagnetic loop operation mode, system transient stability faces certain problems and challenges on several aspects:

- With closed loop, when fault occurs within a relatively short vicinity from a $110 \mathrm{kV}$ substation, due to the influence of earth resistance on distance protection setting, the fault line zone I protection and its adjacent line zone I protection may trip simultaneously. As a result the $110 \mathrm{kV}$ substation would jump all ends from the mains and run into a total loss of main accident.

- If the fault occurs at the busbar of a $110 \mathrm{kVsubstation}$ in which busbar differential protection is usually not installed, the fault can only be removed through zone II distance protection of the opposite side line which takes 1.2 seconds. Thus the scope of the accident may be expanded. Closed loop operation should ensure the system remaining stable under premeditated simulation of accidents.

\section{2) Elastic indexes}

a) Reliability level

There are two main factors affecting the system reliability within electromagnetic looped networks, namely 
the existence of $220 \mathrm{kV}$ single-transformer substations and the existence of low voltage power supply resources.

- If a $220 / 110 \mathrm{kV}$ electromagnetic loop contains $220 \mathrm{kV}$ single-transformer substations, once operated in opened-loop mode, $110 \mathrm{kV}$ busbar would suffer instantaneous loss of voltage and loss all the loads during a $220 \mathrm{kV}$ transformer fault. Power supply to those $110 \mathrm{kV}$ substations can only be restored through the function of automatic bus transfer equipments on opposite side line. While taking the closed-loop operation mode, the $110 \mathrm{kV}$ busbar of the faulted $220 \mathrm{kV}$ substation can be supplied by another substation within the loop, thereby voltage loss situation is avoided. As long as the loads which need to be transferred do not exceed the thermal stability limit of the $110 \mathrm{kV}$ lines, no loads would be shed. Even if the transferred loads exceed the line thermal limit, the amount is effectively reduced by taking premeditated load shedding countermeasures. Therefore the existence of $220 \mathrm{kV}$ singletransformer substation within an electromagnetic loop is crucial to the $110 \mathrm{kV}$ voltage reliability.

- As the automatic bus transfer equipment checks the voltage before operation, if low voltage power supply resources exist in a $220 / 110 \mathrm{kV}$ electromagnetic loop, the resources should be disconnected first. However under closed-loop operation, there is no need for the function of automatic bus transfer thereby no resources would be disconnected. Moreover, during a $220 \mathrm{kV}$ transformer fault, its $110 \mathrm{kV}$ side losses voltage instantaneous for a short term which will cause significant strike to small generators. If the time is long enough, generators would be disconnected from the mains. In fact, it takes 7.5 - 8.0 seconds for the automatic devices to operate thus the disconnection of small generators are inevitable. However in a closed electromagnetic loop, small generators inside network remain reliable and stable. Thereby the closed-loop operation is essential to small generators to stay reliable.

In consideration of the above analysis, the reliability level is divided into four levels as:

- If a $220 \mathrm{kV}$ single-transformer substation exists within the loop, set it to be level I at least, otherwise to be level 0 .

- If $110 \mathrm{kV}$ or below voltage generators exist, set it to be level II.

- If 220kVsingle-transformer substations and low voltage generators are both exist, set it to be level III.

b) Comprehensive economic efficiency

The comprehensive economic efficiency indexes consist of technical reform investment and loss reduction benefit. The comparison is realized via converting these two indexes into annual cost. The loss reduction benefit only considers direct economic loss in this study which is equal to the lost power multiplied by the pool sell price.

Considering practical matters, comprehensive economic efficiency is classified into four levels based on comprehensive annual cost: if the annual cost is less than zero, rate it to be level 0 ; If the annual cost is greater than zero and less or equal to a1, rate it to be level I; If the annual cost is greater than a1 and less or equal to a2, rate it to be level II; If the annual cost is greater than a2, rate it to be level III. The value of a1 and a 2 are determined according to grids enterprise financial evaluation and are counted in units of ten thousands.

\section{EVALUATION PROCESS}

\section{A. Comprehensive evaluation of elastic indexes}

The comprehensive evaluation process of elastic indexes is an important step which decides the final operation mode of an electromagnetic loop. The result is produced via synthetical judging matrix based on reliability indexes and comprehensive economic efficiency indexes as shown in table.1.

TABLE I. COMPREHENSIVE EVALUATION RESULTS OF ELASTIC INDEX

\begin{tabular}{cccccc}
\hline \multirow{2}{*}{$\begin{array}{c}\text { Elastic } \\
\text { indexes }\end{array}$} & \multicolumn{5}{c}{ Comprehensive economic efficiency level } \\
\cline { 2 - 6 } & Level & 0 & I & II & III \\
\hline Reliability & 0 & $\times$ & $\times$ & $\times$ & $\sqrt{ }$ \\
level & I & $\times$ & $\times$ & $\sqrt{ }$ & $\sqrt{ }$ \\
& II & $\times$ & $\sqrt{ }$ & $\sqrt{ }$ & $\sqrt{ }$ \\
& III & $\sqrt{ }$ & $\sqrt{ }$ & $\sqrt{ }$ & $\sqrt{ }$ \\
\hline
\end{tabular}

Note: “ $\sqrt{ }$ " feasible; “ $X$ ” infeasible.

\section{B. Comprehensive evaluation process}

Overall 220/110kV electromagnetic looped network operated under closed-loop mode conforms to four criteria as: no deterioration on system stability, improved reliability, pronounced comprehensive benefit and mature environment for implementation. On the basis of above required criteria, the process to optimize and determine the network operation mode is shown in Fig .2.

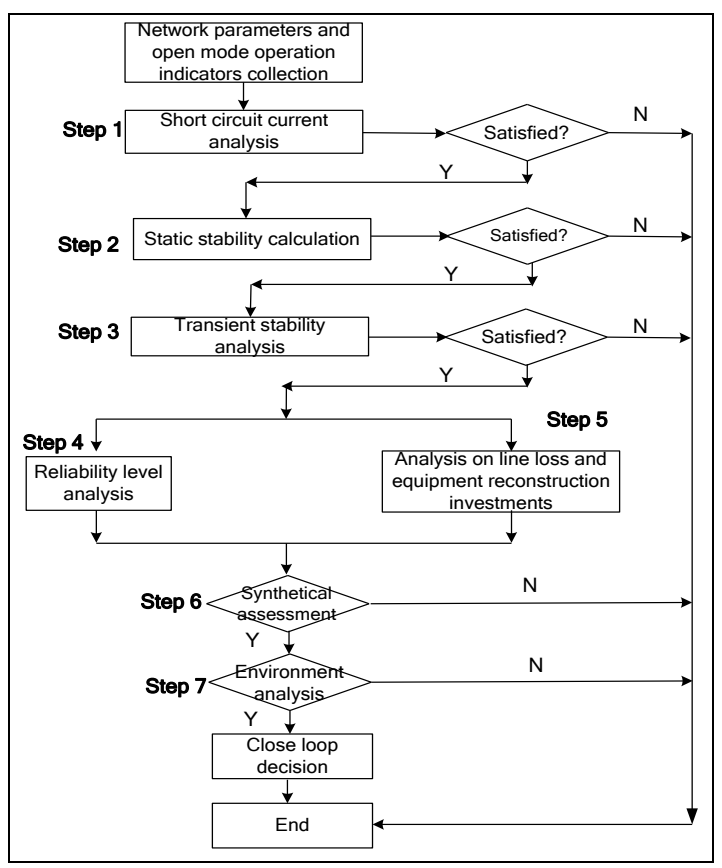

Figure 2. Evaluation process for $220 / 110 \mathrm{kV}$ electromagnetic loop operation modes 
1) Calculate the short circuit currents of involved busbars within the closed loop covering all voltage levels. Compare the results with the rated breaking capacity of every circuit breaker to judge their adequacy.

2) Check N-1 security level under closed operation mode. Focus on the thermal stability of $110 \mathrm{kV}$ lines after a $220 \mathrm{kV}$ line fault or transformer fault. Calculate the closedloop load margin, and compare it with the one under opened-loop condition. Check if the load margin has been improved.

3) Calculate the transient stability of a closed electromagnetic loop. Pay attention to those $110 \mathrm{kV}$ substations in which busbar differential protection is not installed. Verify the stability after busbar fault.

4) Analysis the reliability level of the closed- loop operation mode and compare it with opened- loop operation mode.

5) Analysis the necessities of the protection and communication reconstruction and estimate their investments. Calculate the theoretical line loss under various load levels. Assess general economy considering mentioned aspects.

6) Judge the feasibility of closed-loop operation in respect of reliability level and economic efficiency evaluation.

7) Analysis the maturity of implementation environment which includes equipment maintenance schedule and appropriate precaution measures.

\section{Evaluation Results}

\section{A. Evaluation objects and conditions}

The evaluation process was applied to all $220 / 110 \mathrm{kV}$ electromagnetic looped networks in Hunan system except the ones in Changsha, Zhuzhou and Xiangtan areas due to their intensive configurations which would produce large short circuit currents. Thereby the number of the evaluated and analyzed $220 / 110 \mathrm{kV}$ electromagnetic loops is 98 in total in this study. The proposed process was implemented in Power System Analysis Software Package. The short circuit currents were calculated and analyzed in year of 2015 for Hunan power grid. The value of $a_{1}$ and $a_{2}$ were chosen to be 5 and 10 respectively in this research.

\section{B. Evaluation results}

Based on the above optimization principals, only considering closed loop with single $110 \mathrm{kV}$ tie line situation, the outcome indicated that the reliability level of six loops were declined. The numbers of loops that rated to reliability level 0, level I, level II and level III were 55, 22, 6 and 15 respectively. The numbers of loops that rated as economic efficiency level 0, level I, level II and level III were 40, 20, 17 and 21 respectively. The overall results from elastic evaluation system indicated that it was feasible for 40 electromagnetic loops to be closed in operation, while the 58 rest still infeasible under current situation. Regarding on above indexes, there were 28 loops within Hunan grid can be closed at present.

\section{Implementation effect}

Among the 28 220/110kV electromagnetic loops which can be closed, there were 16 loops rated as reliability level III, 2 loops rated as level II, 6 loops rated as level I and 4 loops rated as level 0 . It verified that closing electromagnetic loop improves the reliability and superiority significantly especially for weak areas where single $220 \mathrm{kV}$ transformer substation exists. Meanwhile the result showed there were 11 loops classified into economic efficiency level III indicating visible economic benefit.

\section{CONCLUSIONS}

1) The impact of $220 / 110 \mathrm{kV}$ electromagnetic loop is relative rather than definite in respect of system secure and economic operation. Therefore whether to open the loop or not should take security, stability, reliability, economy and all other aspects into full consideration.

2) In this paper, the evaluation system is proposed and every evaluation indexes is explicated in detail. The evaluation indexes are divided into two major categories as rigid type and elastic type and their basis and principals are put forward. The evaluation process for open/close mode decision is specified.

3) After applying the proposed evaluation system, it indicated that there were $28220 / 110 \mathrm{kV}$ electromagnetic loops whose closed-mode operation is feasible under current situation.

\section{ACKNOWLEDGMENT}

The authors thank the science and technology project of State Grids in Hunan province (KZ12K16003) for their support.

\section{REFERENCES}

[1] LIN Weifang, SUN Huadong, TANGYong, etal. Analysis and lessons of the blackout in Brazil power grid on November 10, 2009[J]. Automation of Electric Power Systems, 2010, 34(7) : $1-5$.

[2] TANG Siqing, ZHANG Mi, LI Jianshe, etal. Review of blackout in Hainan on September 26th-cause and recommendations $[\mathrm{J}]$. Automation of Electric Power Systems, 2006, 30(1) : 1-7.

[3] YANG Dong, LIU Yutian. Influence of electromagnetic loop in early ultra-high voltage grid[J]. Electric Power Automation Equipment, 2009, 29(6) : 77-80.

[4] HU Yangyu, FU Hongjun, ZHANG Yiming, etal. Analysis on electromagnetically coupled loop operation of tie line connecting Hubei power grid and Henan power grid after building up of Nanyang UHVAC substation[J]. Power System Technology, 2011, 35(6) : 22-26.

[5] LIU Shuyong, GU Qiang, ZHANG Lijuan. Research on power supply scheme based on partitioning of 500/220kV Tianjin power grid during the 11th Five-Year Plan $[\mathrm{J}]$. Power System Technology, 2008, 32(9) : 51-55.

[6] YANG Dong and LIU Yutian . A New Method for Electromagnetic Loop Partitioning Based on Quantitative Analysis of Network Community Structure Characteristics[J]. Automation of Electric Power Systems, 2013, 37(21) : 66-71.

[7] PAN Wei, LIU Wenying, YANG Yihan, etal. Research on operating modes of $750 \mathrm{kV}$ Northwest China power grid electromagnetically coupled with $330 \mathrm{kV}$ power grid[J]. Power System Technology, 2007, 31(15) : 33-38.

[8] ZHANG Zuping, FAN Mingtian, ZHOU Limei. Research of breaking up electromagnetic ring in urban power network $[\mathrm{J}]$. Power System Technology, 2008, 32(19) : 4244.

[9] BAI Hongkun, LI Gansheng. Discussion on weak open-loop mode of electromagnetic loop $[\mathrm{J}]$. Power System Protection and Control, 2010, 38(2) : 60-63.

[10] LIU Mingsong, ZHANG Jian, ZHANG Wenchao. Problems and Countermeasures for Operation and Control of Weak 
Electromagnetic Loops $[\mathrm{J}]$. Automation of Electric Power Systems, 2014, 38(4) : 109-114.

[11] LIU Nan, TANG Xiao-jun, ZHANG Wen-chao, etal. Study on electromagnetic ring opening in Henan province under ultra- high voltage power grid $[\mathrm{J}]$. Power System Protection and Control, 2011, 39(2) : 131-136, 141. 